\title{
SIMULATING PRESSURE DISTRIBUTION ON LENS RELEVANT TO FLUID INJECTION FOR IMMERSION LITHOGRAPHY
}

\author{
*Wenyu Chen, **Xin Fu, **Huayong Yang \\ *The State Key Lab of Fluid Power Transmission and Control \\ Zhejiang University \\ Hangzhou, 086-0571-87953395 China \\ (E-mail: serverlandon@zju.edu.cn) \\ **The State Key Lab of Fluid Power Transmission and Control \\ Zhejiang University \\ Hangzhou, 086-0571-87953395 China
}

\begin{abstract}
Immersion lithography has been proposed as a method for improving optical lithography resolution to $32 \mathrm{~nm}$. The premise behind the concept is to increase the refraction index in the space between the lens and wafer by insertion of a high refractive index liquid in place of the low refractive index air that currently fills the gap. During the scanning and exposure process, immersion liquid is injected into the space between wafer and lens with certain inlet pressure and angle. Because the liquid will act as a lens component during the lithographic process, it must maintain high uniform optical quality. One source of optical degradation may be due to lens distortion caused by the pressure distribution nonuniformity in the fluid flow field. Consequently, any deviations of pressure distribution on flow field boundary in direct contact with lens may damage the uniform optical path.

Three-dimensional computational fluid dynamics models were created to assess the pressure distribution characteristics relevant to flow rates and injecting angles of immersion liquid. Flow field stream patterns were discussed corresponding to dispense port numbers. The numerical simulation results were presented, featuring lens normal and shear pressure and injection flow, considering fluid injecting velocity, dispense ports quantity, and direction angles.
\end{abstract}

\section{KEYWORDS}

Immersion lithography, pressure, fluid flow

\section{INTRODUCTION}

For most of the microelectronics industry history, optical lithography has been the backbone for continuing the trend of making features even smaller. However, as the apparent inability of optical lithography for future requirements, technology evolution to next-generation lithography (NGL) was becoming necessary.
Among all of the competing NGL technologies, Immersion lithography has been proposed in the past as a method to improve the resolution of optical lithography, but more recently it has been gaining popularity due its potential for achieving resolution down to $50 \mathrm{~nm}$ and below. It has shown promise as a technology extending optical lithography without significant changes to the manufacturing infrastructure used for decades. [1,2] The intention of immersion lithography is to increase the 
index of refraction in the space between the lens and wafer by introducing a high refractive index liquid in place of the low refractive index air that currently fills the gap. Because the liquid acts as a lens component during scan-step process, it must maintain a high and uniform optical quality. Thus, an immersion unit structure must be implemented to keep the flow field from leaking. Also, the immersion liquid within the gap has to be updated as substances unwrapped from chemical reacting may affect the optical quality of the liquid. As a result, the streamline patterns in flow field and velocity distribution have to be predicted, and the spatial distribution and magnitude of pressure distribution on lens have been investigated.

\section{FLOW FIELD MODELING AND BASIC INPUT PARAMETERS}

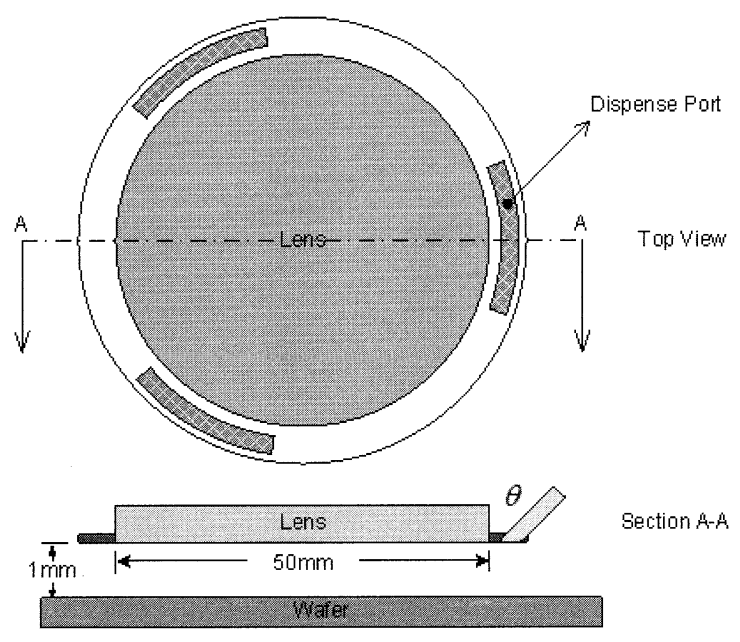

Figure 1. Schematic of the CFD model used to investigate the normal and shear forces on the lens.

A schematic of the model used to study the gap flow field is shown in Figure 1. Top-down and cross-sectional views of the lens/gap/wafer system are shown. To approximate the recovery system, the boundaries at both edges of the model are assumed to have a fixed, negative pressure of $50 \mathrm{~Pa}$. The dispense ports are planar with underneath surface of the lens and the spaces between the lens, dispense port, and liquid collection boundary are enclosed by solid boundaries. The parameter implemented on the above model are listed in Table. 1, and the remaining parameters are discussed in the following context.

Table. 1. Input parameters and material properties for numerical models

\begin{tabular}{|l|l|}
\hline Parameters & Value \\
\hline
\end{tabular}

\begin{tabular}{|c|c|}
\hline Fluid density & $998.2 \mathrm{~kg} / \mathrm{m}^{3}$ \\
\hline Fluid viscosity & $0.001003 \mathrm{~kg} / \mathrm{ms}$ \\
\hline Surrounding pressure & $101325 \mathrm{~Pa}$ \\
\hline Inject velocity & $0.1 \mathrm{~mm} / \mathrm{s}$ \\
\hline Dispense port total area & $55.50 \mathrm{~mm}^{2}$ \\
\hline Gap Thickness & $1 \mathrm{~mm}$ \\
\hline Lens area & $1963.495 \mathrm{~mm}^{2}$ \\
\hline
\end{tabular}

\section{STREAM PATTERNS IN FLOW FIELD}

Through increasing the number of dispense ports, the streamline patterns are listed in Figure 2. Apparently, with asymmetrically ports distribution, the streamline covers entirely and smoothly over the whole lens area, which means better consistency for flow direction. However, taking wafer motion into account, symmetric dispense ports provides much more tolerance of flow change with the course of time due to wafer motion.

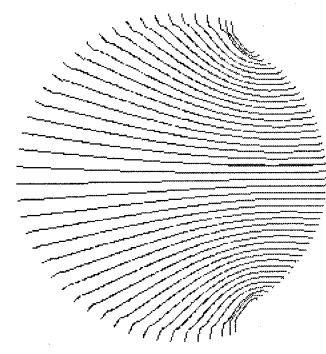

(a) 1 port

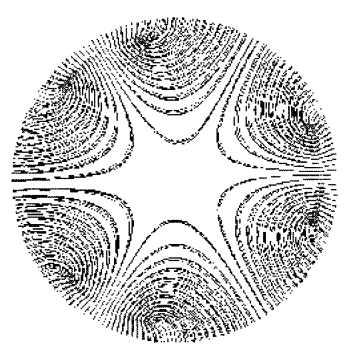

(a) 3 port

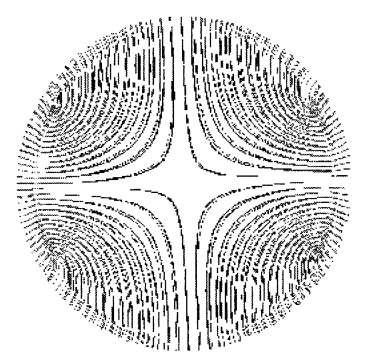

(b) 2 ports

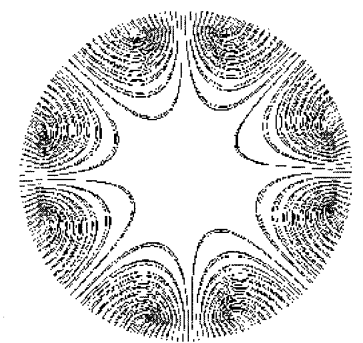

(b) 4 ports
Figure 2. Flow field stream patterns for varying dispense port quantity.

\section{VELOCITY DISTRIBUTION FOR FLOW FIELD FLUID}

The velocity distributions along lens diameter in Section A-A (Figure1.) for varying injecting velocity are presented in Figure 3(a), for varying dispense ports quantity in Figure 3(b), and for varying injecting angle in Figure 3(b). As in Figure 3(a), increasing the injecting velocity partially affects the marginal area of the flow field, and the minimum velocity keeps under $10 \mathrm{~mm} / \mathrm{s}$. As in Figure 3(c), the velocity distribution is left nearly the 
same, which indicates that it's probably not necessary to increase the injection angle by altering the immersion unit structure for higher flowing speed in the fluid field. As in Figure 3(b), the cases with symmetric dispensing geometries have the minimum flowing speed near the lens center, while the case of single dispense port with the maximum and the minimum speed located to the lens margin. This indicates the case of single dispense port endorsed with higher general distribution of flow velocity, which means less updating time for gap fluid field.
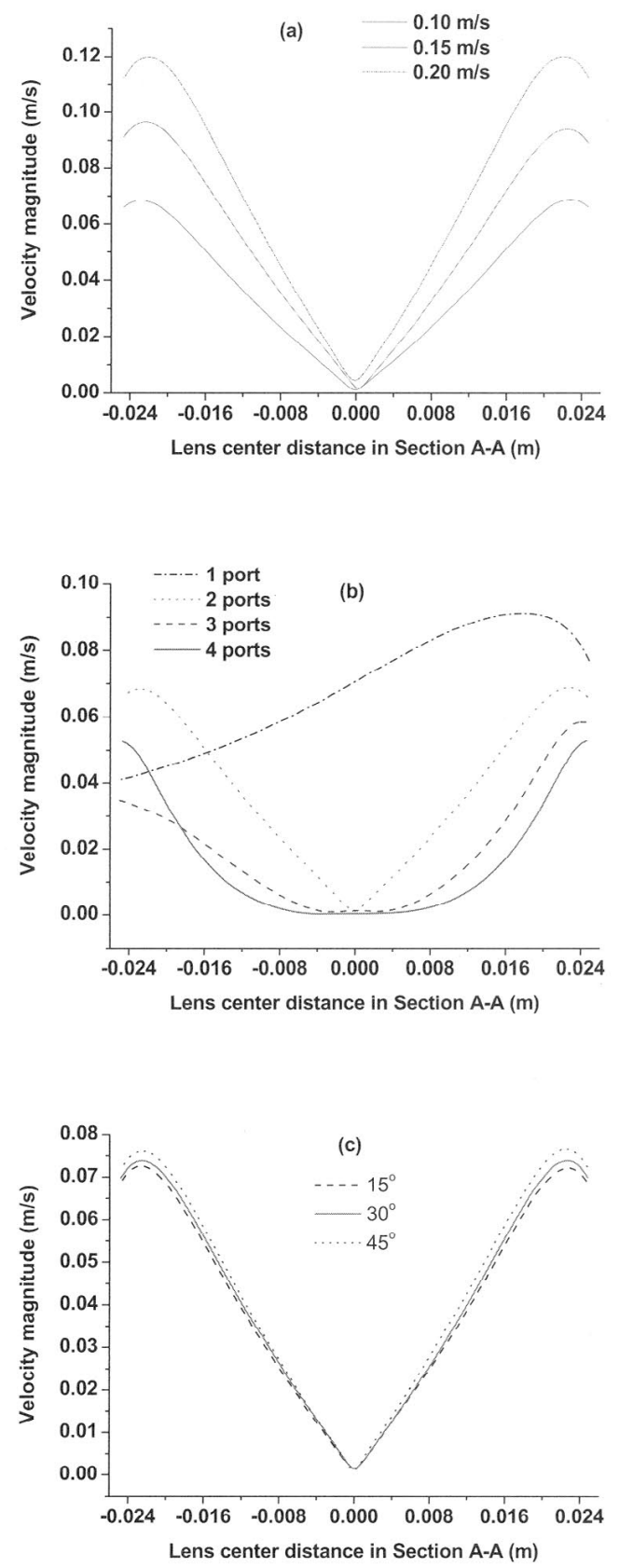

Figure 3. (a) shows the velocity magnitude as a function of lens center distance, for varying injecting velocity. (b) shows the velocity magnitude as a function of lens center distance, for varying dispense ports quantity. (c) shows the velocity magnitude as a function of lens center distance, for varying injecting angle.

\section{NORMAL PRESSURE ON LENS}

With the schematic of the model shown in Figure 1, the shear and normal forces on the lens are studied. Normal forces across the final lens from the immersion flow field are shown in Figure 4, Figure 5, and Figure 6 for various liquid injecting configurations and geometries.

In Figure 4(a), the double dispense ports consist of 60 degree annular sections with a constant velocity at $0.1 \mathrm{~m} / \mathrm{s}$ straight towards the wafer surface, while the velocity has been increased to $0.2 \mathrm{~m} / \mathrm{s}$. For the models in Figure 5(a) and Figure 5(b), which have the same liquid injecting velocity and injecting angles, the dispense port in Figure 5 (b) is divided into four separate parts, symmetrically around the lens area. Since the dispensing ports area remains the same, the total flow rates are equal. The injecting angle has been increased from 15 degree in Figure 6(a) to 45 degree in Figure 6(b), with other parameters being identical.

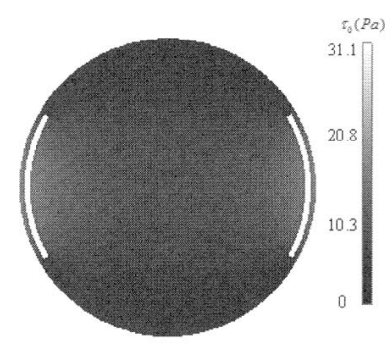

(a) $v=0.1 \mathrm{~m} / \mathrm{s}$

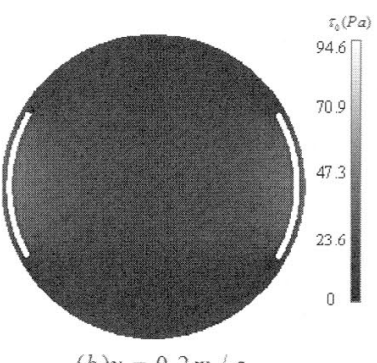

(b) $v=0.2 \mathrm{~m} / \mathrm{s}$
Figure 4. Normal pressure distribution on lens for varying injecting velocity.

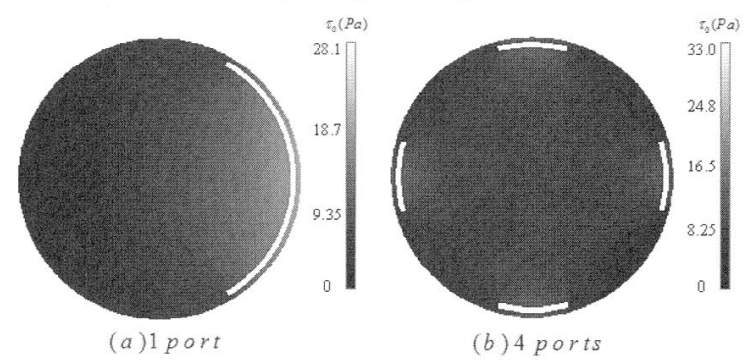

Figure 5. Normal pressure distribution on lens for varying dispense port quantity.

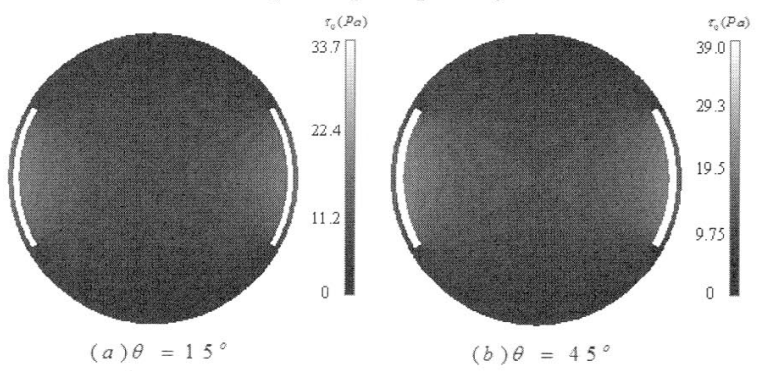

Figure 6. Normal pressure distribution on lens for varying injecting angle. 
In all eight cases, it can be seen that a pressure gradient exists across the lens. The moment resulting from this pressure gradient will tend to cause the lens to tip away from the higher pressure side, which can be avoided by increasing the dispense port number, resulting in symmetric gradient patterns. Normal pressure distribution deviation on lens exacerbates greatly as injecting velocity increases, while not quite affected by more dispense ports or bigger injecting angles. The overall gradient for the lens normal pressure mostly distributes outboard dispense ports opening, which is far away from the lens. However, these forces will have direct impact on wafers and must be investigated further using a structural model of the overall lens housing in order to determine if changes to the immersion unit structure are necessary.

\section{SHEAR PRESSURE ON LENS}

Shear forces across the final lens from the immersion flow field are shown in Figure 7, Figure 8, and Figure 9 for the same liquid injecting configurations and geometries shown in Figure 4, Figure 5, and Figure 6 separately.
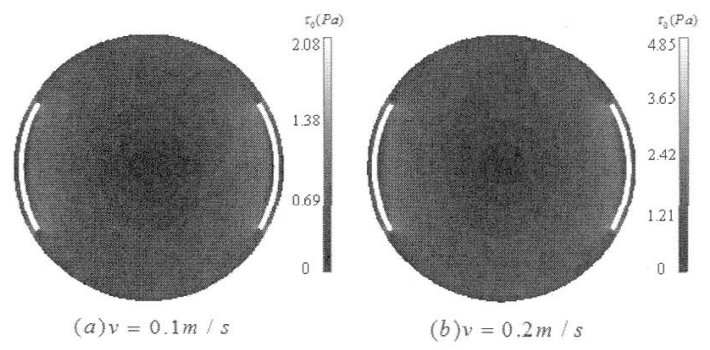

Figure 7. Shear pressure distribution on lens for varying injecting velocity.
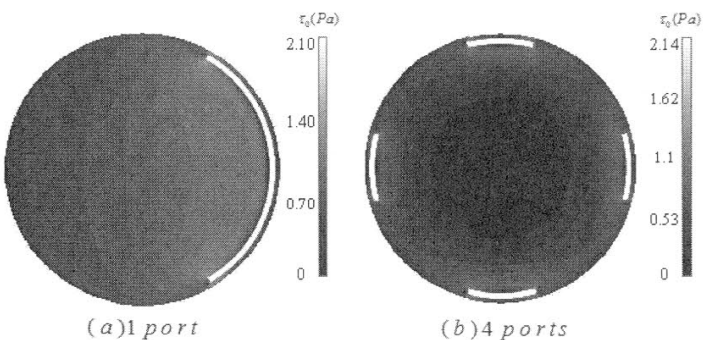

Figure 8. Shear pressure distribution on lens for varying dispense port quantity.

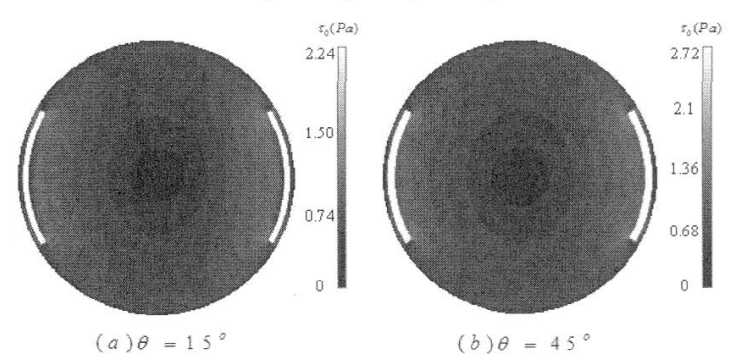

Figure 9. Shear pressure distribution on lens for varying injecting angle.
Shear stresses on the lens may cause birefringence. An analysis has been done to estimate the magnitude of the birefringence induced in the lens. So, the largest birefringence estimates in worst situation would be[3]

$$
\text { birefringence }=2 C R \tau_{0}
$$

where $\mathrm{C}$ is the birefringence constant, $\mathrm{R}$ is the radius of lens, and $\tau_{0}$ is the shear force. Assuming a birefringence constant of $5(\mathrm{~nm} / \mathrm{cm}) /(\mathrm{kg} / \mathrm{cm} 2)$ and using the maximum shear stress obtained from Figure 7(b), the computed birefringence is less than $0.002 \mathrm{~nm}$ for a 5.0 $\mathrm{cm}$ diameter lens. This is a very small value, meaning that the birefringence introduced by shearing pressure will probably not be a factor in imaging defection, relevant to the above eight sets of liquid injecting configurations and geometries.

\section{SUMMARY AND CONCLUSION}

Three-dimensional computational fluid dynamics models have been created to assess the stream patterns in flow field within a range of dispense ports. Velocity distribution for flow field fluid has been discussed with injecting angle, dispense port quantity, and injecting velocity as parameters. The result has indicated that it's probably not necessary to increase the injection angle by altering the immersion unit structure for higher flowing speed in the fluid field and the case of single dispense port is endorsed with higher general distribution of flow velocity, meaning less updating time for gap fluid field. Normal and shear pressure on lens have been presented and discussed. A prediction has been made that the birefringence introduced by shearing pressure will probably not be a factor in imaging defection, relevant to the above eight sets of liquid injecting configurations and geometries simulated.

\section{REFERENCES}

1. M. Switkes, M. Rothschild, J. Microlith. Microfab. Microsyst. 1 (2002) 225.

2. B. Lin, Microelectron. Eng. 6 (1987) 31.

3. A. Wei, Modeling fluid thermomechanical response for immersion lithography scanning. Microelectronic Engineering 73-74 (2004) 29-34 